\title{
Équation de Burgers avec conditions initiales à accroissements indépendants et homogènes
}

\author{
par \\ Laurent CARRARO \\ Département Méthodes et Modèles Mathématiques pour l'Industrie, \\ École des Mines de Saint-Étienne, \\ 158, Cours Fauriel, 42023 Saint-Étienne Cedex 2, France. \\ E-mail: carraro@emse.fr \\ et \\ Jean DUCHON \\ CNRS - URA 740, Université Claude Bernard, \\ 43, Boulevard du 11 novembre 1918, 69622 Villeurbanne Cedex, France.
}

RÉsumé. - On s'intéresse ici aux solutions de l'équation de Burgers non visqueuse avec condition initiale à accroissements indépendants et homogènes, à sauts négatifs. On dégage la notion de solution statistique intrinsèque de cette équation d'évolution et on montre qu'une famille $(X(t) ; t \geq 0)$ de processus de Lévy homogènes est solution statistique intrinsèque de l'équation de Burgers si et seulement si les fonctions exposants $\psi(t, w)$ satisfont l'équation : $\partial_{t} \psi=i \psi \partial_{w} \psi$. L'étude de cette équation permet d'établir l'existence d'une telle solution. Le cas d'une condition initiale brownienne est explicité.

(C) 1998 L'Association Publications de l'Institut Henri Poincaré. Published by Elsevier B.V. All rights reserved

ABSTRACT. - We study here solutions of inviscid Burgers equation with a stochastic initial value with homogeneous and independent increments without positive jumps. We define the notion of intrinsic statistical solution of this evolution equation and show that a family $(X(t) ; t \geq 0)$ of homogeneous Lévy processes is an intrinsic statistical solution of Burgers equation if and only if the exponent functions $\psi(t, w)$ satisfy the differential

AMS Classification: $35 \mathrm{R}, 60 \mathrm{G}, 76 \mathrm{~F}, 35 \mathrm{Q}$.

Annales de l'Institut Henri Poincaré - Analyse non linéaire - 0294-1449

Vol. $15 / 98 / 04 /$

(C) 1998 L'Association Publications de l'Institut Henri Poincaré. Published by Elsevier B.V. All rights reserved 
equation: $\partial_{t} \psi=i \psi \partial_{u} \psi$. The existence of such solutions follows then from the examination of that last equation. The case of a brownian initial condition is made explicit.

(C) 1998 L'Association Publications de l'Institut Henri Poincaré. Published by Elsevier B.V. All rights reserved

\section{INTRODUCTION}

Une approche de la turbulence hydrodynamique consiste à chercher des solutions statistiques (i.e. à donnée initiale aléatoire) des équations régissant le mouvement d'un fluide. L'équation de Burgers :

$$
\partial_{t} u \mid \partial_{x}\left(\begin{array}{l}
1 \\
2
\end{array} u^{2}\right)=c \partial_{x}^{2} u
$$

est considérée dans ce contexte pour son analogie avec celles de NavierStokes $(\varepsilon>0)$ ou d'Euler $(\varepsilon=0)$, malgré bien des différences.

Les solutions de cette équation sont connues explicitement via la transformation de Hopf-Cole (voir [5]). Dans le cas non visqueux ( $\varepsilon=0$ ), cette technique est notamment utilisée pour analyser les solutions lorsque la condition initiale est aléatoire. Par exemple, Sinai [8] s'intéresse au cas d'une condition initiale nulle sur $\mathbb{R}_{-}$et brownienne sur $\mathbb{P}_{+}$. She, Aurell et Frisch [7], à l'aide de calculs numériques, examinent particulièrement des conditions initiales du type browniens fractionnaires. Néanmoins les expressions obtenues se prêtent difficilement à des évaluations de nature probabiliste (moments, densité, loi du processus...).

Formellement, si $u_{0}$ est une condition initiale aléatoire pour laquelle la méthode de Hopf-Cole s'applique, si $u(t,$.$) est la solution de l'équation de$ Burgers avec condition initiale $u_{0}$ et si $\mu_{t}$ désigne la loi de $u(t,$.$) , la famille$ $\left(\mu_{t}\right)_{t}$ de probabilités définies sur un espace $E$ de fonctions réelles d'une variable réelle, vérifie pour toute fonction test $v$ appartenant à l'espace $\mathcal{D}$ des fonctions de classe $C^{\infty}$ à support compact:

$$
\frac{\partial}{\partial t} \int \exp (i\langle u, v\rangle) d \mu_{t}(u)=i \int\left\langle\frac{1}{2} u^{2}, v^{\prime}\right\rangle \exp (i\langle u, v\rangle) d \mu_{t}(u)
$$

Compte tenu de l'invariance galiléenne de l'équation de Burgers (comme de celles d'Euler ou de Navier-Stokes), nous définissons des solutions statistiques " intrinsèques " qui généralisent les solutions homogènes (i.e. invariantes par translation en la variable d'espace $x$ ). 
La première partie introduit les principaux outils probabilistes concernant les processus de Lévy qui scront utiles dans la suitc. Nous définissons alors dans une deuxième partie les solutions statistiques et les solutions statistiques intrinsèques de l'équation de Burgers et montrons comment ces dernières généralisent les solutions statistiques homogènes. La section 3 est consacrée au résultat principal de ce travail, à savoir le lien entre processus de Lévy homogènes de variance finie et solutions statistiques intrinsèques. Cependant, de telles solutions peuvent faire apparaitre des fonctions à sauts positifs, donc ne vérifiant par les conditions d'entropie de Lax. C'est pourquoi nous montrons dans la quatrième partie comment la classe plus restrictive des processus de Lévy homogènes de variance finie à trajectoires dépourvues de sauts positifs est conservée par le flot de l'équation de Burgers et établissons un résultat d'existence et d'unicité de solutions de ce type. Nous explicitons enfin dans la dernière partie les solutions dans le cas d'une condition initiale brownienne et donnons quelques propriétés de celles-ci.

\section{PRÉLIMINAIRES PROBABILISTES}

On commence par préciser le cadre fonctionnel de l'étude qui permet de définir la notion de fonction caractéristique d'un processus aléatoire. On aborde ensuite les propriétés les plus importantes des processus à accroissements indépendants : décomposition de Lévy-Khintchine et mesure de Lévy.

Les processus aléatoires considérés dans la suite sont des processus $X=(X(x))_{x \in \mathbb{R}}$ dont les trajectoires sont cadlag (i.e. sont continues à droite et possèdent une limite à gauche en tout point). Si $E$ désigne l'ensemble des fonctions réelles d'une variable réelle qui sont cadlag, la loi d'un tel processus est la mesure image de la probabilité $P$ sous-jacente par l'application $X . E$ est muni de la tribu cylindrique $\mathrm{C}(E)$ engendrée par les fonctions d'évaluation: $\mathrm{C}(E)=\sigma(\{u \in E \mapsto u(x), x \in \mathbb{R}\})$, choix naturel vis-à-vis du modèle probabiliste. Il est souvent essentiel dans la pratique que cette tribu coïncide avec la tribu borélienne; ce qui n'est pas le cas ici lorsque la topologie de l'espace $E$ est celle de la convergence uniforme (essentiellement car $E$ est alors non séparable). C'est pourquoi on munit cet espace de la métrique de la topologie de Skorokhod sur tout compact, définie comme suit : 
On note, pour $n \in \mathbb{N}^{*}$,

$\Omega_{n}=\{\omega:[-n, n] \rightarrow[-n, n] / \omega$ est bijective, $\omega(-n)=-n, \omega(n)=n\}$.

et si $\omega \in \Omega_{n}$, on pose :

$$
\|\omega\|_{n}=\sup _{\substack{x, y \in[-n, n] \\ x \neq y}}\left|\log \left(\frac{\omega(x)-\omega(y)}{x-y}\right)\right|
$$

On définit ensuite pour $u, v \in E$ :

$$
d(u, v)=\sum_{n \geq 1} 2^{-n} \operatorname{Min}\left(1, d_{n}(u, v)\right),
$$

où

$$
\begin{array}{r}
d_{n}(u, v)=\operatorname{Inf}\left\{\varepsilon>0, \exists \omega \in \Omega_{n},\|\omega\|_{n} \leq \varepsilon\right. \\
\left.\sup _{x \in[-n, n]}|u(x)-v(\omega(x))| \leq \varepsilon\right\} .
\end{array}
$$

On montre que muni de cette métrique, $E$ est un espace polonais (voir Billingsley [2]); ce qui implique en particulier la coïncidence des tribus borélienne et cylindrique.

On désigne par $M$ l'espace des mesures de Radon sur $\mathbb{R}$, à support compact et par $D$ le sous-espace vectoriel engendré par les masses de Dirac. Dans la suite, pour simplifier l'écriture, si $\lambda$ désigne la mesure de Lebesgue sur $\mathbb{R}$, on identifiera une fonction $v$ appartenant à l'espace $\mathcal{D}$ des fonctions de classe $C^{\infty}$ à support compact à la mesure $v . \lambda$ et on écrira par exemple $v(A)$, si $A$ est un borélien de $\mathbb{R}$.

Avant d'indiquer la dualité naturelle entre $E$ et $M$, il est nécessaire de rappeler quelques propriétés des fonctions de $E$. Ainsi, toute fonction de $E$ est borélienne, localement bornée et l'ensemble de ses points de discontinuité est au plus dénombrable. Par suite, il est loisible de poser, pour $u \in E$ et $\nu \in M$ :

$$
\langle u, \nu\rangle=\int u(x) d \nu(x) .
$$

L'application :. $u \in E \mapsto\langle u, \nu\rangle$ va nous intéresser particulièrement dans ce qui suit. Il est bon de noter que cette application n'est pas continue en général (elle ne l'est pas lorsque $\nu \in D$ ); d'où l'intérêt du :

LEMmE 1. - (i) Si $v \in \mathcal{D}, u \in E \mapsto\langle u, v\rangle$ est continue.

(ii) Si $\nu \in M$, il existe une suite $\left(v_{n}\right)_{n}$ de fonctions de $\mathcal{D}$ telle que :

$$
\left\{\begin{array}{l}
\forall u \in E,\langle u, \nu\rangle=\lim _{n}\left\langle u, v_{n}\right\rangle \\
\forall n, v_{n}(\mathbb{R})=\nu(\mathbb{R})
\end{array}\right.
$$


Preuve. - (i) Si $u_{n} \underset{n}{\rightarrow} u, u_{n}(x) \underset{n}{\rightarrow} u(x)$ en tout point $x$ de continuité de $u$ (voir [2]). Comme de plus, la suite $u_{n}(x)$ est bornée uniformément (en $x \in \operatorname{Supp} v$ et $n \in \mathbb{N}$ ), on déduit du théorème de convergence dominée la convergence de $\left\langle u_{n}, v\right\rangle$ vers $\langle u, v\rangle$.

(ii) Fixons $\rho \in \mathcal{D}$ telle que $\int \rho(x) d x=1$ et $\operatorname{Supp} \rho \subset \mathbb{R}_{+}$; puis posons, pour $n \in \mathbb{N}^{*}, \rho_{n}(x)=n \rho(n x)$. Il est alors clair que les fonctions $v_{n}=\rho_{n} * \nu$ sont éléments de l'espace $\mathcal{D}$, et si $u \in E$, on a :

$$
\begin{aligned}
\left\langle u, v_{n}\right) & =\int u(x) \int \rho_{n}(x-y) d \nu(y) d x \\
& =\int\left(\int u(x) \rho_{n}(x-y) d x\right) d \nu(y) .
\end{aligned}
$$

Le théorème de Fubini est ici applicable par $\rho_{n}$ et $\nu$ sont à support compact.

De plus, par continuité à droite de $u$,

$$
\int u(x) \rho_{n}(x-y) d x=\int_{0}^{+\infty} u(y+z) \rho_{n}(z) d z \underset{n}{\rightarrow} u(y) .
$$

Comme par ailleurs,

$\left|\int u(x) \rho_{n}(x-y) d x\right| \leq \operatorname{Sup}\{|u(x)|, x \in \operatorname{Supp} \nu+\operatorname{Supp} \rho\} \in L^{1}(|\nu|)$,

le théorème de convergence dominée s'applique et fournit le résultat.

On déduit immédiatement de ce lemme que pour toute mesure $\nu$ de $M$, l'application :

$$
u \in E \mapsto\langle u, \nu\rangle
$$

est mesurable; ce qui permet de donner un sens à la :

DÉfinition 1. - Soit $\mu$ une probabilité sur E muni de la tribu $\mathrm{C}(\mathrm{E})$; on appelle fonction caractéristique de $\mu$ l'application $\hat{\mu}$ définie sur $M$ par:

$$
\hat{\mu}(\nu)=\int_{E} e^{i\langle u, \nu\rangle} d \mu(u)
$$

Cette fonction détermine la mesure $\mu$; plus précisément :

LEMME 2. - Une probabilité $\mu$ sur $(E, \mathrm{C}(\mathrm{E}))$ est caractérisée par :

(i) $\hat{\mu}(\nu)$, pour $\nu \in D$,

ou par:

(ii) $\hat{\mu}(v)$, pour $v \in \mathcal{D}$. 
Preuve. - Le premier point est conséquence de l'injectivité de la transformation de Fourier des probabilités sur $\mathbb{R}^{n}\left(n \in \mathbb{N}^{*}\right)$ et du théorème d'unicité du prolongement d'une probabilité du $\pi$-système engendré par les évaluations $u \in E \mapsto u(x), x \in \mathbb{R}$, à la tribu engendrée $\mathrm{C}(\mathrm{E})$. Le point (ii) est conséquence immédiate du point (i) et du point (ii) du lemme 1.

Nous ne considérons dans ce qui suit que la loi des accroissements des processus étudiés; il faut pour cela donner quelques notations et résultats analogues à ce qui précède.

On définit tout d'abord la tribu $\mathrm{C}^{\prime}(E)=\sigma(\{u \in E \mapsto u(x)-$ $u(y) ; x, y \in \mathbb{R})\}$ engendrée par les accroissements et on note :

$$
\begin{gathered}
M_{0}=\{\nu \in M, \nu(\mathbb{R})=0\}, D_{0}=\{\nu \in D, \nu(\mathbb{R})=0\}, \\
\mathcal{D}_{0}=\{v \in \mathcal{D}, v(\mathbb{R})=0\} .
\end{gathered}
$$

On peut remarquer que lorsque $\nu \in M_{0}$, l'application $u \in E \mapsto\langle u, \nu\rangle$ est $\mathrm{C}^{\prime}(E)$-mesurable; ce qui permet de considérer la fonction caractéristique d'une probabilité $\mu$ sur $\left(E, \mathrm{C}^{\prime}(E)\right)$ comme une fonction définie sur $M_{0}$ par la formule (1). Cette fonction mérite toujours son nom puisque :

LEMME 3. - Une probabilité $\mu$ sur $\left(E, \mathrm{C}^{\prime}(\mathrm{E})\right)$ est caractérisée par :

(i) $\hat{\mu}(\nu)$, pour $\nu \in D_{0}$, ou par:

(ii) $\hat{\mu}(v)$, pour $v \in \mathcal{D}_{0}$.

Preuve. - Identique à celle du lemme 2.

Terminons ces préliminaires fonctionnels en rappclant le résultat suivant, qui a trait à la différentiabilité au sens de Gâteaux de la fonction caractéristique $\hat{\mu}$ :

LEMME 4. - Soit $\mu$ une probabilité sur $(E, \mathrm{C}(\mathrm{E}))$. Alors, $\hat{\mu}$ est de classe $C^{2}$ sur $M$ si et seulement si $\forall \nu \in M, \int|\langle u, \nu\rangle|^{2} d \mu(u)<+\infty$; dans ce cas, si $\nu, \nu_{1}, \nu_{2}$ sont éléments de $M$, on $a$ :

$$
D^{2} \hat{\mu}(\nu)\left(\nu_{1}, \nu_{1}\right)=-\int\left\langle u, \nu_{1}\right\rangle\left\langle u, \nu_{2}\right\rangle \exp (i\langle u, \nu\rangle) d \mu(u) .
$$

Preuve. - L'implication $\left(\forall \nu \in M, \int|\langle u, \nu\rangle|^{2} d \mu(u)<+\infty \Rightarrow \hat{\mu}\right.$ est de classe $\left.C^{2}\right)$ est immédiate. L'implication inverse résulte de la considération, pour $\nu \in M$, de la probabilité $\mu_{\nu}$ image de $\mu$ par l'application : $u \in E \mapsto\langle u, \nu\rangle$. Comme $\hat{\mu}_{\nu}$ est de classe $C^{2}$, on a $\int_{\mathbb{B}} t^{2} d \mu_{\nu}(t)<+\infty$; c'est-à-dire $\int|\langle u, \nu\rangle|^{2} d \mu(u)<+\infty$. 
Remarquons que ce lemme reste valable pour une probabilité $\mu$ sur $\left(E, \mathrm{C}^{\prime}(E)\right)$ à condition de s'intéresser à la différentiabilité de $\hat{\mu}$ sur l'espace $M_{0}$.

Avant d'aborder les notions fondamentales concernant les processus à accroissements indépendants, il est bon de donner l'interprétation probabiliste des précédentes notions.

On appellera processus aléatoire à trajectoires dans $E$ toute application mesurable $X:(\Omega, \mathcal{F}) \rightarrow(E, \mathrm{C}(E))$; la loi d'un tel processus est alors la mesure image de la probabilité $P$ sur $\mathcal{F}$ par l'application $X$. En d'autres termes, si $\mu$ est cette loi, on a :

$$
\forall B \in \mathrm{C}(E), P(X \in B)=\mu(B) .
$$

De façon analogue, la loi des accroissements du processus $X$ est la trace de la probabilité $\mu$ sur la tribu $\mathrm{C}^{\prime}(E)$. Les lemmes 2 et 3 donnent alors des caractérisations de la loi du processus ou de ses accroissements.

DÉfinition 2. - (i) Un processus $X=(X(x))_{x \in \mathbb{R}}$ est dit à accroissements indépendants (en abrégé PAI) si pour tout $n \in N(n \geq 3)$ et tous $x_{1}<x_{2}<\ldots<x_{n}$ les variables aléatoires $X\left(x_{2}\right)-X\left(x_{1}\right)$, $X\left(x_{3}\right)-X\left(x_{2}\right) \ldots, X\left(x_{n}\right)-X\left(x_{n-1}\right)$ sont indépendantes.

(ii) Le PAI $X=(X(x))_{x \in \mathbb{R}}$ est dit à accroissements indépendants et homogènes (en abrégé PAIH) si pour tous $x<y$, les variables $X(y)-X(x)$ et $X(y-x)-X(0)$ ont même loi.

(iii) Un processus $X$ est un processus de Lévy (resp. de Lévy homogène) si $X$ est un PAI (resp. PAIII), à trajectoires dans $E$, et continu en probabilité, i.e. vérifiant :

$$
\forall \varepsilon>0, P(|X(y) \quad X(x)|>\varepsilon)_{y-x \rightarrow 0}^{,} 0 .
$$

Si $X=(X(x))_{x \in \mathbb{R}}$ est un processus de Lévy homogène, sa fonction caractéristique admet une expression simple. On a en effet, pour $y>x$ et $w \in \mathbb{R}$ :

$$
E[\exp \{i w(X(y)-X(x))\}]=\exp \{(y-x) \psi(w)\},
$$

où $\psi: \mathbb{R} \rightarrow \mathbb{C}$ est continue et vérifie $\psi(0)=0$. Cette dernière fonction, appelée exposant de Lévy, est définie de façon unique par $X$ (ou plus exactement par la loi des accroissements de $X$ ), on notera donc $\psi=\psi_{X}$. Notons que réciproquement, la donnée de la fonction $\psi$ détermine la loi des accroissements du processus $X$ puisque la formule (3) se généralise au cas des mesures de $M_{0}$ en :

$$
\forall \nu \in M_{0}, \hat{\mu}(\nu)=\exp \left\{\int \psi(\nu([x,+\infty[)) d x\} .\right.
$$


Cette fonction $\psi$ admet une décomposition, dite de Lévy-Khintchine, qui est le reflet de la décomposition du processus $X$ en somme de ses sauts et d'un processus à trajectoires continues.

De façon précise, notons, pour $A \in \mathcal{B}\left(\mathbb{R} \times \mathbb{R}^{*}\right)$ et $\omega \in \Omega$ :

$$
N(A)(\omega)=\operatorname{Card}\left\{(x, s) \in \mathbb{R} \times \mathbb{R}^{*}, X(x)(\omega)-X\left(x^{-}\right)(\omega)=s\right\}
$$

et

$$
\Lambda(A)=E[N(A)] .
$$

Proposition-Définition. - (i) $N$, appelée mesure des sauts $d u$ processus $X$, est une mesure de Poisson aléatoire d'intensité $\Lambda$, i.e.:

- $\forall A \in \mathcal{B}\left(\mathbb{R} \times \mathbb{R}^{*}\right), N(A)<+\infty \Rightarrow N(A)$ est une variable aléatoire de Poisson d'espérance $\Lambda(A)$.

- Pour presque tout $\omega, N().(\omega)$ est une mesure positive localement finie sur $\mathbb{R} \times \mathbb{R}^{*}$.

- Pour toute suite $A_{1}, \ldots, A_{n}$ de parties disjointes de $\mathcal{B}\left(\mathbb{R} \times \mathbb{R}^{*}\right)$, les variables aléatoires $N\left(A_{1}\right), \ldots, N\left(A_{n}\right)$ sont indépendantes.

(ii) $\Lambda$ est une mesure positive sur $\mathbb{R} \times \mathbb{R}^{*}$, produit de la mesure de Lebesgue sur $\mathbb{R}$ par une mesure, notée $n$ et appelée mesure de Lévy du processus $X$, sur $\mathbb{R}^{*}$ qui vérifie :

$$
\int_{\mathbb{R}^{\star}} \min \left(s^{2}, 1\right) n(d s)<+\infty .
$$

Théorème De Paul Lévy ([1], [4]). - Soit $X$ un processus de Lévy homogène et $N$ sa mesure des sauts. Alors, si $x<y$, on a :

$$
\begin{aligned}
X(y)-X(x)= & a(B(y)-B(x))+\tilde{b}(y-x) \\
& +\int_{|s|>1} s N([x, y], d s) \\
& +\int_{|s| \leq 1} s\{N([x, y], d s)-(y-x) n(d s)\} .
\end{aligned}
$$

où $a \geq 0, \tilde{b} \in \mathbb{R}$ et $B$ est un processus dont la loi est celle du mouvement brownien standard.

De plus, les processus $B$ et $X-B$ sont indépendants.

Notons seulement à propos de cette décomposition que l'expression attendue ne devrait a priori pas comporter d'intégrale en $n$; l'apparition 
de celle-ci est due aux problèmes de convergence de l'intégrale en $N$ au voisinage de 0 (i.e. au cumul de petits sauts).

Il découle simplement de cette décomposition la forme de l'exposant de Lévy du processus $X$ :

Corollaire 1. - Sous les hypothèses précédentes, on a la formule de Lévy-Khintchine :

$$
\begin{aligned}
\psi(w)= & -\frac{a^{2}}{2} w^{2}+i \tilde{b} w+\int_{|s|>1}\left(e^{i s w}-1\right) n(d s) \\
& +\int_{|s| \leq 1}\left(e^{i s w}-1-i s w\right) n(d s)
\end{aligned}
$$

Remarquons que cette écriture caractérise les exposants de Lévy des processus de Lévy homogènes au sens où si $\psi: \mathbb{R} \rightarrow \mathbb{C}$ vérifie (6), il existe un processus de Lévy $X$ dont $\psi$ est l'exposant.

Les processus que nous considérons par la suite seront toujours de carré intégrable; d'où l'intérêt du :

Lemme 5. - Soit $X$ un processus de Lévy homogène de mesure de Lévy $n$; alors :

$$
\forall x<y, X(y)-X(x) \in L^{2} \Leftrightarrow \int_{\mathbb{R}^{*}} s^{2} n(d s)<+\infty .
$$

Dans la suite, lorsque la condition du lemme 5 est satisfaite, on parlera pour simplifier de processus de Lévy homogène de variance finie. Dans ce cas, du fait des propriétés d'intégrabilité de la mesure $n$, la formule de Lévy-Khintchine se simplifie :

Corollaire 2. - Soit $\psi$ l'exposant de Lévy d'un processus de Lévy homogène de variance finie; alors :

$$
\psi(w)=-\frac{a^{2}}{2} w^{2}+i \hat{b} w+\int_{\mathbb{R}^{*}}\left(e^{i s w}-1-i s w\right) n(d s)
$$

LEMME 6. - Soit $\psi: \mathbb{R} \rightarrow \mathbb{C}$. $\psi$ est l'exposant de Lévy d'un processus de Lévy homogène de variance finie si et seulement si $\psi(0)=0, \psi$ est de classe $C^{2}$ et $-\psi^{\prime \prime}$ est définie positive.

Preuve. - Soit $X$ un processus de Lévy homogène, on sait que $\psi_{X}$ vérifie $\psi_{X}(0)=0$. Si de plus $X$ est de variance finie, la condition $\int_{\mathbb{R}^{*}} s^{2} n(d s)<+\infty$ permet d'appliquer le théorème de dérivabilité sous le signe intégral; on montre ainsi que $\psi_{X}$ est de classe $C^{2}$ et que 
$-\psi_{X}^{\prime \prime}(w)=a^{2}+\int_{\mathbb{R}^{*}} t^{i s w} s^{2} n(d s)$. La fonction $-\psi_{X}^{\prime \prime}$, transformée de Fourier de la mesure positive finie $m(d s)=a^{2} \delta_{0}(d s)+s^{2} n(d s)$, est donc définie positive.

Réciproquement, si $\psi$ satisfait les conditions du lemme, le théorème de Bochner fournit l'existence d'une mesure $m$ sur $\mathbb{R}$ dont $-\psi^{\prime \prime}$ est la transformée de Fourier. En isolant la masse éventuelle de $\mu$ en 0 , on peut toujours écrire :

$$
m(d s)=a^{2} \delta_{0}(d s)+s^{2} n(d s),
$$

où $n$ est une mesure positive sur $\mathbb{R}^{*}$ telle que $\int_{\mathbb{R}^{*}} s^{2} n(d s)<+\infty$.

En intégrant deux fois l'équation :

$$
-\psi^{\prime \prime}(w)=a^{2}+\int_{\mathbb{R}^{*}} e^{i s w^{\prime}} s^{2} n(d s),
$$

on obtient immédiatement l'identité (7), où $\hat{b}=-i \psi^{\prime}(0)$.

\section{SOLUTIONS STATISTIQUES INTRINSÈQUES}

Commençons par établir formellement la notion de solution statistique. Si $X_{0}$ est un processus aléatoire et si $X(t, x)$ est la solution de l'équation de Burgers au temps $t$, à l'abscisse $x$ avec condition initiale $X_{0}$, la famille $(X(t, x))_{t \geq 0, x \in \mathbb{R}}$ est un processus aléatoire à deux indices $t$ et $x$. Nous allons voir qu'alors, la loi du processus $X(t)$ défini par $X(t)(x)=X(t, x)$ suit une équation d'évolution simple. Une telle approche ne permettra évidemment pas d'obtenir des renseignements probabilistes du type loi conjointe des processus $X(s)$ et $X(t)$ en des instants distincts $s$ et $t$ mais est susceptible d'aider à la connaissance des lois des processus $X(t), t>0$.

En raisonnant donc de façon formelle, nous obtenons à partir de l'équation de Burgers non visqueuse l'équation, valable pour toute fonction $v$ de $\mathcal{D}$ :

$$
\begin{aligned}
& \int_{\Omega} \int_{\mathbb{R}} \partial_{t} X(t, x) v(x) d x \exp (i\langle X(t, .), v\rangle) d P(\omega) \\
& =-\int_{\Omega} \int_{\mathbb{R}} \partial_{x}\left[\frac{1}{2} X(t, x)^{2}\right] v(x) d x \exp (i\langle X(t, .), v\rangle) d P(\omega),
\end{aligned}
$$

soit :

$$
\begin{aligned}
& \int_{\Omega} \frac{1}{i} \frac{\partial}{\partial t} \exp (i\langle X(t, .), v\rangle) d P(\omega) \\
& \quad=\int_{\Omega} \int_{\mathbb{R}} \frac{1}{2} X(t, x)^{2} v^{\prime}(x) d x \exp (i\langle X(t, .), v\rangle) d P(\omega),
\end{aligned}
$$


soit encore, en notant $\mu_{t}$ la loi du processus $X(t)$ :

$$
\frac{\partial}{\partial_{t}} \hat{\mu}_{t}(v)=i \int_{E}\left\langle\frac{1}{2} u^{2}, v^{\prime}\right\rangle \exp (i\langle u, v\rangle) d \mu_{t}(u)
$$

d'où :

DÉFINITION 3. - Une solution statistique de l'équation de Burgers est une famille $\left(\mu_{t}\right)_{t \geq 0}$ de probabilités sur $(E, \mathrm{C}(\mathrm{E}))$ vérifiant :

$$
\forall v \in \mathcal{D}, \quad \frac{\partial}{\partial t} \hat{\mu}_{t}(v)=i \int_{E}\left\langle\frac{1}{2} u^{2}, v^{\prime}\right\rangle \exp (i\langle u, v\rangle) d \mu_{t}(u)
$$

Comme nous l'avons vu plus haut, la probabilité bien connue dans le cas d'un processus de Lévy est la loi des accroissements du processus. Or, si $v \in \mathcal{D}_{0}, \hat{\mu}_{t}(v)$ est bien définie par la trace de $\mu_{t}$ sur $\mathrm{C}^{\prime}(\mathrm{E})$ mais par contre, l'application : $u \in E \mapsto\left\langle\frac{1}{2} u^{2}, v^{\prime}\right\rangle \exp (i\langle u, v\rangle)$ n'est pas $\mathrm{C}^{\prime}(\mathrm{E})-$ mesurable. Ce qui va nous mener à modifier la définition.

Pour $u \in E$, nous désignerons, pour $a<b$, par $\langle u\rangle_{a, b}$ la moyenne de la fonction $u$ sur l'intervalle [a, b] :

$$
\langle u\rangle_{a, b}=\frac{1}{b-a} \int_{a}^{b} u(x) d x .
$$

DÉFINITION 4. - Une solution statistique intrinsèque de l'équation de Burgers est une famille $\left(\mu_{t}\right)_{t \geq 0}$ de probabilités sur $\left(E, \mathrm{C}^{\prime}(\mathrm{E})\right)$ vérifiant :

$$
\begin{gathered}
\forall v \in \mathcal{D}_{0}, \\
\frac{\partial}{\partial t} \hat{\mu}_{t}(v)=\lim _{\substack{a \rightarrow-\infty \\
b \rightarrow+\infty}} i \int_{E}\left\langle\frac{1}{2}\left(u-\langle u\rangle_{a, b}\right)^{2}, v^{\prime}\right\rangle \exp (i\langle u, v\rangle) d \mu_{t}(u)
\end{gathered}
$$

Notons au passage que la définition précédente est cohérente car la fonction : $u \in E \mapsto\left\langle\frac{1}{2}\left(u-\langle u\rangle_{a, b}\right)^{2}, v^{\prime}\right\rangle \exp (i\langle u, v\rangle)$ est $\mathrm{C}^{\prime}(\mathrm{E})$ mesurable.

Comme nous l'annoncions dans l'introduction, il est important de noter, à l'appui de notre définition, le rapport entre les deux définitions précédentes.

Rappelons pour cela qu'un processus $X=(X(x))_{x \in R}$ à trajectoires dans $E$ est un processus homogène si pour tout $y \in \mathbb{R}$, les processus $X(X(x))_{x \in \mathbf{R}}$ et $(X(x+y))_{x \in \mathbf{R}}$ sont de même loi. 
Proposition 1. - Soit X un processus homogène, dont le moment d'ordre 2 est fini, on $a$ :

$$
\begin{gathered}
\lim _{\substack{a \rightarrow-\infty \\
b \rightarrow+\infty}} i \int\left\langle\frac{1}{2}\left(u-\langle u\rangle_{a, b}\right)^{2}, v^{\prime}\right\rangle \exp (i\langle u, v\rangle) d \mu(u) \\
=i \int\left\langle\frac{1}{2} u^{2}, v^{\prime}\right\rangle \exp (i\langle u, v\rangle) d \mu(u) .
\end{gathered}
$$

Preuve. - En développant le carré du terme de gauche de la formule (10), il suffit de montrer que :

$$
A(a, b)+B(a, b) \underset{\substack{a \rightarrow-\infty \\ b \rightarrow+\infty}}{\rightarrow \rightarrow} 0
$$

où :

$$
\begin{aligned}
& A(a, b)=\int\langle u\rangle_{a, b}\left\langle u, v^{\prime}\right\rangle \exp (i\langle u, v\rangle) d \mu(u) \\
& B(a, b)=\int\langle u\rangle_{a, b}^{2}\left\langle 1, v^{\prime}\right\rangle \exp (i\langle u, v\rangle) d \mu(u) .
\end{aligned}
$$

Comme $v$ est à support compact, il est évident que le terme $B(a, b)$ est nul; il suffit donc de s'intéresser à $A(a, b)$. Or, d'après le théorème ergodique, si $\mathcal{J}$ désigne la sous-tribu de $\mathrm{C}(\mathrm{E})$ des événements invariants par translation, on a :

$$
\langle u\rangle_{a, b} \underset{\substack{a \rightarrow-\infty \\ b \rightarrow+\infty}}{\rightarrow} U(u(0) / \mathcal{J})
$$

où $E(. / \mathcal{J})$ désigne l'espérance conditionelle relativement à la sous-tribu $\mathcal{J}$ et où la convergence a lieu presque sûrement et dans $L^{2}(\mu)$.

Par ailleurs, la finitude du moment d'ordre 2 indique que l'application : $u \mapsto\left\langle u, v^{\prime}\right\rangle$ appartient à $L^{2}(\mu)$; par suite,

$$
A(a, b) \underset{\substack{a \rightarrow-\infty \\ b \rightarrow+\infty}}{\rightarrow} \int E(u(0) / \mathcal{J})\left\langle u, v^{\prime}\right\rangle \exp (i\langle u, v\rangle) d \mu(u) .
$$

Il suffit donc de voir, pour achever la preuve, que cette expression limite, notée $A$, est nulle. Comme $E$ est un espace polonais, il existe une version régulière de la loi conditionnelle de $u$ par rapport à $E(u(0) / \mathcal{J})=y$. En notant $\mu_{y}$ cette loi conditionnelle et $\nu$ la loi de $E(u(0) / \mathcal{J})$, on a donc :

$$
A=\int_{\mathbb{R}} \int_{E}\left\langle u, v^{\prime}\right\rangle \exp (i\langle u, v\rangle) d \mu_{y}(u) d \nu(y)
$$


Or la probabilité $\mu_{y}$ est la loi d'un processus homogène (et ergodique) dont lc moment d'ordre 2 est fini. La conclusion sera donc acquise si l'on montre que pour toute loi $\mu_{0}$ d'un processus homogène de variance finie, on a :

$$
\int_{E}\left\langle u, v^{\prime}\right\rangle \exp (i\langle u, v\rangle) d \mu_{0}(u)=0 .
$$

La preuve formelle de cette identité est très simple : si, pour $v \in \mathcal{D}$, on considère l'application $T: x \in \mathbb{R} \mapsto \hat{\mu}_{0}\left(\tau_{x} v\right)$, où $\tau_{x}$ désigne la translation par $x$, la dérivée de cette application est donnée par la formule :

$$
T^{\prime}(x)=i \int_{E}\left\langle u, \tau_{x} v^{\prime}\right\rangle \exp \left(i\left\langle u, \tau_{x} v\right\rangle\right) d \mu_{0}(u) .
$$

En particulier, si le processus considéré est homogène, l'application $T$ est constante et sa dérivée en 0 est donc nulle; ce qui fournit (13).

Pour justifier la dérivation précédente, on utilise l'inégalité $\mid \exp (i a)-$ $1-i a \mid \leq \frac{a^{2}}{2}$, valable pour $a$ réel. Flle fournit ici, pour $h \neq 0$ :

$$
\begin{aligned}
& \frac{1}{h}\left|\int_{E}\left(e^{i\left\langle u, \tau_{h} v\right\rangle}-e^{i\langle u, v\rangle}-i\left\langle u, \tau_{h} v-v\right\rangle e^{i\langle u, v\rangle}\right) d \mu_{0}(u)\right| \\
& \quad \leq \frac{h}{2} \int_{E}\left\langle u, \frac{1}{h}\left(\tau_{h} v-v\right)\right\rangle \exp (i\langle u, v\rangle) d \mu_{0}(u)
\end{aligned}
$$

et l'hypothèse faite sur $\mu_{0}$, i.e. $\int_{E} u(x)^{2} d \mu_{0}(u)<+\infty$, montre que cette dernière quantité tend vers 0 lorsque $h$ tend vers 0 . Une dernière utilisation de cette même hypothèse montre enfin que :

$$
\int_{E} i\left\langle u, \frac{1}{h}\left(\tau_{h} v-v\right)\right\rangle e^{i\langle u, v\rangle} d \mu_{0}(u) \underset{h \rightarrow 0}{\rightarrow} \int_{E}\left\langle u, v^{\prime}\right\rangle e^{i\langle u, v\rangle} d \mu_{0}(u)
$$

ce qui termine la preuve.

\section{SOLUTIONS STATISTIQUES INTRINSÈQUES ET PROCESSUS DE LÉVY}

Le résultat qui suit montre de quelle façon la famille des processus de Lévy est conservée par le flot de l'équation de Burgers.

THÉORÈme 1. - Soit $(X(t) ; t \geq 0)$ une famille de processus de Lévy homogènes. On désigne par $\mu_{t}$ la loi des accroissement de $X(t)$ et on note $\psi(t,$.$) l'exposant de Lévy du processus X(t)$. On suppose que : 
- (H1) Pour tout $t \geq 0, \psi(t,$.$) est de classe C^{2}$.

- (H2) Pour tout réel $w, \psi(., w)$ est dérivable et $\partial_{t} \psi$ est localement bornée.

Alors, la famille $\left(\mu_{t}\right)_{t \geq 0}$ est solution statistique intrinsèque de l'équation de Burgers si et seulement si :

$$
\partial_{t} \psi=i \psi \partial_{w} \psi
$$

Notons, avant d'aborder la démonstration de ce résultat, que, en vertu du lemme 6, supposer la condition (H1) satisfaite revient à considérer que pour tout $t \geq 0$, le PAIH $X(t)$ est de variance finie.

Preuve. - Nous poserons pour simplifier les écritures, pour $v \in \mathcal{D}_{0}$ et $x \in \mathbb{R}, w(x)=v([x,+\infty[)$. Commençons par expliciter le terme de gauche de l'identité (9): on fixe pour cela $0<t_{0}<t_{1}$. Pour tout réel $x$, la fonction : $t \in] 0,+\infty[\mapsto \psi(t, w(x))$ est dérivable. De plus, l'hypothèse $(\mathrm{H} 2)$ indique que la fonction $\partial_{t} \psi$ est bornée sur le compact $\left[t_{0}, t_{1}\right] \times w(\mathrm{Suppv})$ et le théorème de dérivation sous le signe intégral fournit ainsi, pour $t$ appartenant à $] t_{0}, t_{1}[$ :

$$
\frac{\partial}{\partial t} \int \psi(t, w(x)) d x=\int \partial_{t} \psi(t, w(x)) d x .
$$

Cette formule, liée à la relation (4), donne alors :

$$
\frac{\partial}{\partial t} \hat{\mu}_{l}(v)-\hat{\mu}_{t}(v) \int \partial_{w} \psi(t, w(x)) d x
$$

L'évaluation du terme de droite de (9) est beaucoup plus délicate; nous utiliserons pour cela le :

LEMme 7. - Soit $X$ un processus de Lévy homogène d'exposant de Lévy $\psi$ de classe $C^{2}$. Alors, $\hat{\mu}$ est deux fois différentiable sur $M_{U}$ et, $\forall \nu, \nu_{1}$, $\nu_{2} \in M_{0}$ :

$$
\begin{aligned}
& D \hat{\mu}(\nu)\left(\nu_{1}\right)=\left(\int \nu _ { 1 } \left(\left[x,+\infty[) \psi^{\prime}(\nu([x,+\infty[)) d x) \hat{\mu}(\nu) .\right.\right.\right. \\
& D^{2} \hat{\mu}(\nu)\left(\nu_{1}, \nu_{2}\right) \\
& =\left\{\int \nu _ { 1 } \left(\left[x,+\infty[) \nu_{2}\left(\left[x,+\infty[) \psi^{\prime \prime}(\nu([x,+\infty[))\right.\right.\right.\right.\right. \\
& \quad+\left(\int \nu _ { 1 } \left(\left[x,+\infty[) \psi^{\prime}(\nu[x,+\infty[)) d x)\right.\right.\right. \\
& \quad \times\left(\int \nu _ { 2 } \left(\left[x,+\infty[) \psi^{\prime}(\nu[x,+\infty[)) d x)\right\} \hat{\mu}(\nu) .\right.\right.
\end{aligned}
$$


Preuve. - La formule (4) montre que pour obtenir (16), on est amené à étudier la dérivabilité en 0 de la fonction :

$$
h \in \mathbb{R} \mapsto \int \psi\left(\nu \left(\left[x,+\infty[)+h \nu_{1}([x,+\infty[) d x .\right.\right.\right.
$$

Or, lorsque $h$ appartient à un voisinage compact de 0 , comme $\psi^{\prime}$ est bornée sur tout compact, on a :

$$
\begin{aligned}
& \mid \frac{\partial}{\partial h} \psi\left(\nu \left(\left[x,+\infty[)+h \nu_{1}([x,+\infty[) \mid\right.\right.\right. \\
& \quad=\mid \nu_{1}\left(\left[x,+\infty[) \psi^{\prime}\left(\nu \left(\left[x,+\infty[)+h \nu_{1}([x,+\infty[)) \mid\right.\right.\right.\right.\right. \\
& \quad \leq M 1_{\text {Supp } \nu_{1}}(x) .
\end{aligned}
$$

La formule (16) est alors une conséquence immédiate du théorème de dérivabilité sous le signe intégral et de l'identité (4).

Le même raisonnement est valable pour obtenir (17); il suffit de considérer dans ce cas l'application :

$$
h \in \mathbb{R} \mapsto \int \nu_{1}\left(\left[x,+\infty[) \psi^{\prime}\left(\nu \left(\left[x,+\infty[)+h \nu_{2}([x,+\infty[)) d x\right.\right.\right.\right.\right.
$$

qui, du fait que $\psi^{\prime \prime}$ est localement bornée, est dérivable en 0 .

Fin de la preuve du théorème 1. - Nous noterons désormais $\rho=\frac{1}{b-a} 1_{[a, b]}$ et utiliserons dans la démonstration qui suit uniquement le fait que lorsque $a \rightarrow-\infty$ et $b \rightarrow+\infty$, les probabilités $\rho$. $\lambda$ convergent faiblement vers 0 .

Évaluons, pour $v$ élément de $D_{0}$, le terme de droite de (9), c'est-à-dire :

$$
A(\rho)=i \int_{E}\left\langle\frac{1}{2}(u-\langle u, \rho\rangle)^{2}, v^{\prime}\right\rangle \exp (i\langle u, v\rangle) d \mu_{t}(u) .
$$

Nous allons appliquer le théorème de Fubini; notons pour cela la majoration, valable pour tout réel $x$ :

$$
\begin{gathered}
\left|\frac{1}{2}(u(x)-\langle u, \rho\rangle)^{2} v^{\prime}(x) \exp (i\langle u, v\rangle)\right| \\
\leq M 1_{\text {Suppv }}(x)(u(x)-\langle u, \rho\rangle)^{2} .
\end{gathered}
$$

De plus, en utilisant le lemme 4 , on a :

$$
\int_{E}(u(x)-\langle u, \rho\rangle)^{2} d \mu_{t}(u)=-D^{2} \hat{\mu}_{t}(0)\left(\nu_{x, p} \nu_{x, \rho}\right),
$$

où $\nu_{x, \rho}$ est la mesure $d\left(\nu_{x, \rho}\right)(y)=d \delta_{x}(y)-\rho(y) d y$. 
La relation (17) fournit alors :

$$
\begin{aligned}
\int_{E}(u(x)-\langle u, \rho\rangle)^{2} d \mu_{t}(u)= & -\int \nu_{x, \rho}\left(\left[y,+\infty[)^{2} \partial_{u}^{2} \psi(t, 0) d y\right.\right. \\
& -\left(\int \nu _ { x , \rho } \left(\left[y,+\infty[) \partial_{w} \psi(t, 0) d y\right)^{2}\right.\right. \\
\leq & -\int \nu_{x, \rho}\left(\left[y,+\infty[)^{2} \partial_{w}^{2} \psi(t, 0) d y\right.\right.
\end{aligned}
$$

Or, $\mid \nu_{x, \rho}\left(\left[y,+\infty[) \mid 21_{K}(y)\right.\right.$, où $K=\operatorname{Supp} \rho \cup \operatorname{Supp} v$. Par suite,

$$
\int_{E}(u(x)-\langle u, \rho\rangle)^{2} d \mu_{t}(u) \leq-4 \lambda(K) \partial_{w}^{2} \psi(t, 0) .
$$

On déduit de (19) et (20) que la fonction :

$$
(x, u) \mapsto \frac{1}{2}(u(x)-\langle u, \rho\rangle)^{2} v^{\prime}(x) \exp (i\langle u, v\rangle)
$$

est $d \lambda \otimes d \mu_{t}-$ intégrable et le théorème de Fubini mène à :

$$
A(\rho)=\frac{i}{2} \int\left(\int_{E}\left\langle u, \nu_{x, \rho}\right\rangle^{2} \exp (i\langle u, v\rangle) d \mu_{t}(u)\right) v^{\prime}(x) d x
$$

soit encore, en utilisant à nouveau le lemme 4 :

$$
A(\rho)=-\frac{i}{2} \int D^{2} \hat{\mu}_{t}(v)\left(\nu_{x, \rho} \nu_{x, \rho}\right) v^{\prime}(x) d x .
$$

L'utilisation de la relation (17) permet d'aboutir à :

$$
A(\rho)=\hat{\mu}_{t}(v)(B(\rho)+C(\rho))
$$

avec :

(22) $B(\rho)=-\frac{i}{2} \int\left(\int \nu_{x, \rho}\left(\left[y,+\infty[)^{2} \partial_{w}^{2} \psi(t, w(y)) d y\right) v^{\prime}(x) d x\right.\right.$

(23) $C(\rho)=-\frac{i}{2} \int\left(\int \nu_{x, \rho}([y,+\infty]) \partial_{w} \psi(t, w(y)) d y\right)^{2} v^{\prime}(x) d x$ 
Explicitons tout d'abord $B(\rho)$ :

$$
\begin{aligned}
B(\rho)= & -\frac{i}{2} \int\left(\int_{-\infty}^{x}\left(\int_{-\infty}^{y} \rho(z) d z\right)^{2} \cdot \partial_{w}^{2} \psi(t, w(y)) d y\right) v^{\prime}(x) d x \\
& -\frac{i}{2} \int\left(\int_{x}^{+\infty}\left(-\int_{y}^{+\infty} \rho(z) d z\right)^{2} \partial_{w}^{2} \psi(t, w(y)) d y\right) v^{\prime}(x) d x
\end{aligned}
$$

soit, après intégration par parties (les termes tout intégrés s'évanouissent du fait que $v$ est élément de $\mathcal{D}$ ) :

$$
\begin{aligned}
B(\rho)= & \frac{i}{2} \int\left(\int_{-\infty}^{x} \rho(z) d z\right)^{2} \partial_{w}^{2} \psi(t, w(x)) v(x) d x \\
& -\frac{i}{2} \int\left(\int_{x}^{+\infty} \rho(z) d z\right)^{2} \partial_{w}^{2} \psi(t, w(x)) v(x) d x
\end{aligned}
$$

Mais comme $\rho(\mathbb{R})=1$, on a :

$$
\left(\int_{-\infty}^{x} \rho(z) d z\right)^{2}-\left(\int_{x}^{+\infty} \rho(z) d z\right)^{2}=\int_{-\infty}^{x} \rho(z) d z-\int_{x}^{+\infty} \rho(z) d z .
$$

Par suite,

$$
\left.B(\rho)=\frac{i}{2} \int\left(\int_{-\infty}^{x} \rho(z) d z-\int_{x}^{+\infty} \rho(z) d z\right) \partial_{w}^{2} \psi(t, w(x)) v(x) d x\right)
$$

Après avoir noté que $\partial_{w}^{2} \psi(t, w(x)) v(x)=-\frac{\partial}{\partial x} \partial_{w} \psi(t, w(x))$, on intègre une deuxième fois par parties; nous obtenons ainsi :

$$
\begin{aligned}
B(\rho)= & i \int\left(\partial_{w} \psi(t, w(x))-\partial_{w} \psi(t, 0)\right) \rho(x) d x \\
- & {\left[\left(\int_{-\infty}^{x} \rho(z) d z-\int_{x}^{+\infty} \rho(z) d z\right)\right.} \\
& \left.\times\left(\partial_{w} \psi(t, w(x))-\partial_{w} \psi(t, 0)\right)\right]_{-\infty}^{+\infty} .
\end{aligned}
$$

Comme la fonction : $x \mapsto \partial_{w} \psi(t, w(x))-\partial_{w} \psi(t, 0)$ est continue et nulle à l'infinie, la convergence faible de $\rho$ vers 0 montre que :

$$
B(\rho) \rightarrow 0 .
$$

Vol. 15, $n^{\circ} 4-1998$ 
En ce qui concerne le terme $C(\rho)$, le procédé est analogue à celui utilisé pour $B(\rho)$; la formule (23) mène à :

$$
C(\rho)=-\frac{i}{2} \int I(x)^{2} v^{\prime}(x) d x
$$

où :

$$
\begin{aligned}
I(x)= & \int_{-\infty}^{x}\left(\int_{-\infty}^{y} \rho(z) d z\right) \partial_{w} \psi(t, w(y)) d y \\
& +\int_{x}^{+\infty}\left(\int_{y}^{+\infty} \rho(z) d z\right) \partial_{w} \psi(t, w(y)) d y
\end{aligned}
$$

On obtient alors par intégration par parties :

$$
C(\rho)=i \int I(x) I^{\prime}(x) v(x) d x
$$

Or,

$$
\begin{aligned}
I^{\prime}(x)= & \left(\int_{-\infty}^{x} \rho(z) d z\right) \partial_{w} \psi(t, w(x)) \\
& +\left(\int_{r}^{+\infty} \rho(z) d z\right) \partial_{w} \psi(t, w(x))=\partial_{w} \psi(t, w(x))
\end{aligned}
$$

donc $C(\rho)=i \int I(x) \partial_{w} \psi(\ell, w(x)) v(x) d x$.

De plus, $\partial_{w} \psi(t, w(x)) v(x)=-\frac{\partial}{\partial x} \psi(t, w(x))$; comme par ailleurs, la fonction $\psi(t, w()$.$) tend vers 0$ à l'infini, on obtient après une nouvelle intégration par parties:

$$
C(\rho)=i \int I^{\prime}(x) \psi(t, w(x)) d x
$$

soit :

$$
C(\rho)=i \int \partial_{w} \psi(t, w(x)) \psi(t, w(x)) d x
$$

En conclusion, les formules (15), (18), (21), (24) et (25) montrent que la famille $\left(\mu_{t}\right)_{t \geq 0}$ est solution statistique intrinsèque de l'équation de Burgers si et seulement si :

$$
\begin{gathered}
\forall w \in \mathcal{D} \\
\int \partial_{t} \psi(t, w(x)) d x=i \int \partial_{w} \psi(t, w(x)) \psi(t, w(x)) d x
\end{gathered}
$$


Nous terminerons la démonstration à l'aide du :

Lemme 8. - Soit $f: \mathbb{B} \rightarrow \mathbb{C}$ borélienne, localement bornée, telle que $f(0)=0$; alors :

$$
f=0 \Leftrightarrow \forall w \in \mathcal{D}, \quad \int f(w(x)) d x=0 .
$$

Preuve. - Fixons un réel non nul $w$; nous supposerons $w>0$ pour fixer les idées. Pour $\varepsilon>0$, on construit $w_{\varepsilon}$ élement de $\mathcal{D}$ tel que :

$$
\left\{\begin{array}{l}
\operatorname{Supp} w_{\varepsilon}=[0,1] \\
w_{\varepsilon}(x)=w \text { si } x \in[\varepsilon, 1-\varepsilon] \\
\forall x \in \mathbb{R}, w_{\varepsilon}(x) \in[0, w]
\end{array}\right.
$$

On a alors :

$$
0=\int f\left(w_{\varepsilon}(x)\right) d x=(1-2 \varepsilon) f(w)+\int_{[0, \varepsilon] u[1-\varepsilon, 1]} f(w(x)) d x
$$

Si on note $M$ la bornée supérieure de $|f|$ sur l'intervalle $[0, w]$, on obtient :

$$
\left|\int_{[0, \varepsilon] u[1-\varepsilon, 1]} f(w(x)) d x\right| \leq 2 M \varepsilon .
$$

Le résultal du lemme en découle.

On achève la preuve du théorème en appliquant le lemme à la fonction $f=\partial_{t} \psi-i \psi \partial_{w} \psi$, qui s'annule en 0 .

Notons que le théorème précédent ne donne ni l'existence, ni l'unicité de solution statistiques intrinsèques de l'équation de Burgers et que les conditions d'entropie de Lax ne sont pas prises en compte. Dans notre contexte, ces conditions imposent à la solution de l'équation de Burgers d'avoir des sauts tous négatifs. Nous allons donc considérer maintenant des processus de Lévy homogènes de variance finie à sauts négatifs.

\section{SOLUTIONS STATISTIQUES INTRINSÈQUES ET PKOCESSUS DE LÉVY À SAUTS NÉGATIFS}

Nous allons donner tout d'abord très succintement les propriétés des processus de Lévy à sauts négatifs qui seront utiles dans la suite. Avant tout, il convient de bien noter que ce que nous appelons processus à sauts négatifs n'impose pas au processus considéré d'avoir des sauts mais lui interdit d'avoir des sauts positifs.

Vol. $15, \mathrm{n}^{\circ} 4-1998$. 
Rappelons que la mesure de Lévy d'un processus de Lévy de variance finie à sauts négatifs vérifie la condition :

$$
\int_{]-\infty, 0[} s^{2} n(d s)<+\infty
$$

et permet d'écrire la formule de Lévy-Khintchine sous la forme :

$$
\psi(w)=-\frac{a^{2}}{2} w^{2}+i \hat{b} w+\int_{]-\infty, 0[}\left(e^{i s w}-1-i s w\right) n(d s)
$$

Dans ce cas, en posant, pour $\omega \in \Pi^{+}=\{\omega \in C, \operatorname{Re}(\omega)>0\}$ :

$$
\varphi(w)=\frac{a^{2}}{2} w^{2}+\hat{b} w+\int_{]-\infty, 0[}\left(e^{s \omega}-1-s \omega\right) n(d s)
$$

on définit une fonction analytique sur $\Pi^{+}$qui se prolonge continûment à $\overline{\Pi^{+}}$, et :

$$
\psi(w)=\varphi(i w), \text { si } w \in \mathbb{R}
$$

Nous appelerons dans ce qui suit second exposant de Lévy du processus $X$ la restriction de la fonction $\varphi$ à $\mathbb{R}_{+}(\varphi$ est appelé exposant de Laplace dans [1]).

LEMME 9. - La loi des accroissements d'un processus de Lévy homogène de variance finie à sauts négatifs $X$ est déterminée par son second exposant.

Preuve. - Si $\varphi$ est connue sur $\mathbb{R}_{+}$, elle l'est également sur $\Pi^{+}$par prolongement analytique, puis sur $\overline{\Pi^{+}}$par prolongement par continuité. La formule (28) montre alors que $\psi$ est également connue; ce qui suffit pour déterminer la loi des accroissements de $X$ d'après la formule (4) et le lemme 3. Réciproquement, la donnée de $\psi$ détermine, en vertu du principe de symétrie de Schwarz, la fonction $\varphi$ sur $\Pi^{+}$, donc en particulier sur $\mathbb{R}_{+}$.

Le précédent résultat d'injectivité est complété par le lemme suivant, qui a trait à la surjectivité :

LEMME 10. - Une fonction $\varphi: \mathbb{R}_{+} \rightarrow \mathbb{R}$ est le second exposant de Lévy d'un processus de Lévy homogène de variance finie et à sauts négatifs si et seulement si $\varphi$ est de classe $C^{\infty}$ sur $] 0,+\infty\left[\right.$, de classe $C^{2}$ sur $[0,+\infty[$. $\varphi(0)=0$, et $\varphi^{\prime \prime}$ est complètement monotone.

Preuve. - Nous ne la reproduisons pas; elle est très analogue à celle du lemme 6. Rappelons seulement qu'une fonction $f: \mathbb{R}_{+} \rightarrow \mathbb{R}$ est complètement monotone si elle est de la forme :

$$
f(\omega)=\int_{\mathbf{R}_{-}} e^{s \omega} m(d s)
$$


où $m$ est une mesure positive finie sur $\mathbb{R}_{-}$.

Le théorème de Bernstein caractérise cette classe de fonctions par la condition nécessaire et suffisante :

$f$ est continue sur $\mathbb{R}_{+}$, de classe $C^{\infty}$ sur $] 0,+\infty[$ et vérifie :

$$
\forall n \in \mathbb{N},(-1)^{n} f(n) \geq 0 .
$$

Remarquons que le résultat du lemme 9 peut égalcment êtrc ćtabli à l'aide de la formule suivante, valable pour toute fonction $\omega$ positive, appartenant à $\mathcal{D}$ :

$$
\int_{E^{\prime}} e^{\left\langle u, \omega^{\prime}\right\rangle} d \mu(u)=E\left[\exp \left(\left\langle X, \omega^{\prime}\right\rangle\right)\right]=\exp \left(\int \varphi(\omega(x)) d x\right)
$$

Cette formule permet en effet de connaître la loi $\mu$ de $X$ sur $\left(E, \mathrm{C}^{\prime}(\mathrm{E})\right)$. Il suffit pour cela d'établir un lemme analogue au lemme 3 , qui utilise la transformation de Laplace au lieu de la transformation de Fourier.

Terminons ces préliminaires en évoquant le cas des processus de Lévy de variance finie, à sauts négatifs et dont les trajectoires sont à variation bornée sur tous les compacts de $\mathbb{R}$; nous dirons pour simplifier qu'ils sont à variation bornée. Nous les rencontrerons comme solutions de l'équation de Burgers. Ils sont caractérisés par une forme nouvelle de la formule de Lévy-Khintchine (27) :

$$
\varphi(\omega)=b \omega+\int_{]-\infty, 0[}\left(e^{s \omega}-1\right) n(d s),
$$

où

$$
\int_{]-\infty, 0[} \max \left(|s|, s^{2}\right) n(d s)<+\infty
$$

qui traduit au mieux la décomposition du processus $X$ en somme de ses sauts :

$$
X(y)-X(x)=b(y-x)+\int_{]-\infty, 0[} s N([x, y], d s)
$$

Cette formule permet également une interprétation simple du coefficient $b$ : il s'agit d'un coefficient de dérive (linéaire).

THÉORÈme 2. - Soit $(X(t) ; t \geq 0)$ une famille de processus de Lévy homogènes de variance finie et à sauts négatifs. On désigne par $\mu_{t}$ la loi des accroissements de $X(t)$ et on note $\varphi(t,$.$) le second exposant de Lévy$ du processus $X(t)$. On suppose que :

- $\left(\mathrm{H}^{\prime} 2\right)$ Pour tout $\omega \geq 0, \varphi(., \omega)$ est dérivable et $\partial_{t} \varphi$ est localement bornée. 
Alors, la famille $\left(\mu_{t}\right)_{t \geq 0}$ est solution statistique intrinsèque de l'équation de Burgers si et seulement si $\varphi$ est solution de l'équation de Burgers :

$$
\left\{\begin{array}{l}
\partial_{t} \varphi+\varphi \partial_{\omega} \varphi=0 \\
t>0, \quad \omega>0
\end{array}\right.
$$

Preuve. - Nous ne la reproduisons pas; il suffit de répéter la preuve du théorème 1 en remplaçant chaque fois que de besoin la transformation de Fourier par celle de Laplace.

Nous allons terminer cette section avec un dernier théorème qui donne l'existence et l'unicité de telles solutions statistiques intrinsèques à accroissements inépendants et homogènes à sauts négatifs (i.e. vérifiant les conditions d'entropie).

Théorìme 3. - Soit $\varphi_{0}$ le second exposant de lévy d'un processus de Iévy homogène de variance finie et à sauts négatifs de loi $\mu_{0}$. On suppose que :

$$
\varphi_{0}^{\prime}(0) \geq 0
$$

Il existe alors une unique solution $\varphi$ à l'équation de Burgers (31) avec condition initiale $\varphi_{0}$. De plus, pour tout $t>0$, la fonction $\varphi(t,$.$) est le$ second exposant de Lévy d'un processus de Lévy homogène de variance finie, à sauts négatifs, à variation bornée, de loi $\mu_{t}$.

Enfin, la famille $\left(\mu_{t}\right)_{t \geq 0}$ est l'unique solution statistique intrinsèque de l'équation de Burgers formée de processus de Lévy homogènes de variance finie, à sauts négatifs, vérifiant $\left(\mathrm{H}^{\prime} 2\right)$, avec condition initiale $\mu_{0}$.

Remarque. - La condition (H3) signifie que le processus de Lévy $X(0)$ de loi $\mu_{0}$ vérifie $E(X(0)(y)-X(0)(x)) \geq 0$, pour $y>x$; elle fournit la non explosion de la solution en temps fini.

Preuve. - Déjà, du fait que $\varphi_{0}^{\prime \prime}$ est positive et que $\varphi_{0}^{\prime}(0) \geq 0$, on en déduit que la fonction $\varphi_{0}^{\prime}$ est positive. Par suite, pour tout $t>0$, la fonction :

$$
\omega \in \mathbb{R}_{+} \mapsto \omega+t \varphi_{0}(\omega) \in \mathbb{R}_{+}
$$

est une bijection et un $C^{\infty}$-difféomorphisme de $] 0,+\infty[$ sur $] 0,+\infty[$; on notera l'application réciproque $h(t,$.$) . On en déduit aisément l'existence$ et l'unicité d'une solution (régulière) à l'équation (31) : elle est donnée par la formule :

$$
\varphi\left(t, \omega+t \varphi_{0}(\omega)\right)=\varphi_{0}(\omega),
$$


soit :

$$
\varphi(t, \omega)=\varphi_{0}(h(t, \omega))
$$

Montrons maintenant que $\varphi(t,$.$) est le second exposant de Lévy d'un$ processus de Lévy homogène de variance finie et à sauts négatifs : il suffit, en vertu du lemme 10 , de vérifier que $\varphi$ est de classe $C^{2}$ sur $[0,+\infty[$ et que $\partial_{\omega}^{2} \varphi(t,$.$) est complètement monotone.$

On tire déjà de la formule (33) les deux identités :

$$
\begin{gathered}
\partial_{\omega} \varphi\left(t, \omega+t \varphi_{0}(\omega)\right)=\frac{\varphi_{0}^{\prime}(\omega)}{1+t \varphi_{0}^{\prime}(\omega)} \\
\partial_{\omega}^{2} \varphi\left(t, \omega+t \varphi_{0}(\omega)\right)=\frac{\varphi_{0}^{\prime \prime}(\omega)}{\left(1+t \varphi_{0}^{\prime}(\omega)\right)^{3}} .
\end{gathered}
$$

On en déduit immédiatement que $\varphi$ est de classe $C^{2}$ sur $[0,+\infty[$ et que $\partial_{\omega}^{2} \varphi$ est positive. Pour établir la complète monotonie de $\partial_{\omega}^{2} \varphi$ nous utiliserons la caractérisation de Bernstein donnée au lemme 10. Il s'agit de prouver par récurrence que :

$$
\forall n \geq 2,(-1)^{n} \partial_{\omega}^{n} \varphi \geq 0 .
$$

La propriété est vrai à l'ordre 2; supposons-la satisfaite pour les entiers compris entre 2 et $n-1$. En dérivant $n$ fois (par rapport à la variable $\omega$ ) l'équation (31), on obtient :

$$
\partial_{t} \partial_{\omega}^{n} \varphi+\partial_{\omega}^{n+1}\left(\frac{1}{2} \varphi^{2}\right)=0
$$

soit, à l'aide de la formulc de Lcibniz :

$$
\partial_{t} \partial_{\omega}^{n} \varphi+\varphi \partial_{\omega}^{n+1} \varphi+(n+1) \partial_{\omega} \varphi \partial_{\omega}^{n} \varphi=-\frac{1}{2} \sum_{k=2}^{n_{b}-1}\left(\begin{array}{c}
n+1 \\
k
\end{array}\right) \partial_{\omega}^{k} \varphi \partial_{\omega}^{n+1-k} \varphi
$$

L'hypothèse de récurrence montre alors que :

$$
(-1)^{n}\left[\partial_{t} \partial_{\omega}^{n} \varphi+\varphi \partial_{\omega}^{n+1} \varphi+(n+1) \partial_{\omega} \varphi \partial_{\omega}^{n} \varphi\right] \geq 0
$$

Appliquons cette inégalité en $\left(t, \omega+t \varphi_{0}(\omega)\right)$; en utilisant la relation (33), on aboutit à :

$$
\begin{aligned}
& \frac{d}{d t}\left[(-1)^{n} \partial_{\omega}^{n} \varphi\left(t, \omega+t \varphi_{0}(\omega)\right)\right] \\
& \quad+(n+1) \partial_{\omega} \varphi\left(t, \omega+t \varphi_{0}(\omega)\right)(-1)^{n} \partial_{\omega}^{n} \varphi\left(l, \omega+\iota \varphi_{0}(\omega)\right) \geq 0
\end{aligned}
$$


c'est-à-dire à une inégalité différentielle de la forme $\frac{d u}{d t}+f u \geq 0$ (ici, $\left.j=\frac{\varphi_{0}^{\prime}}{1+t \varphi_{0}^{\prime}}\right)$. Compte tenu de la condition initiale $u(0) \geq 0$, on en déduit que pour tout $t, u(t) \geq 0$; autrement dit $(-1)^{n} \partial_{\omega}^{n} \varphi\left(t, \omega+t \varphi_{0}(\omega)\right) \geq 0$. Ce qui suffit à établir la complète monotonie de $\varphi^{\prime \prime}$.

Il nous reste à voir que l'hypothèse $\left(\mathrm{H}^{\prime} 2\right)$ est satisfaite et que les trajectoires du processus de loi $\mu_{t}$ sont, pour $t>0$, à variation bornée.

Pour obtenir $\left(\mathrm{H}^{\prime} 2\right)$, il suffit de vérifier que $\varphi \partial_{\omega} \varphi$ est localement bornée. Or,

$$
\varphi \partial_{\omega} \varphi(t, \omega)=\varphi_{0}(h(t, \omega)) \frac{\varphi_{0}^{\prime}(h(t, \omega))}{1+t \varphi_{0}^{\prime}(h(t, \omega))} ;
$$

le résultat en découle.

Il reste à voir que le processus de Lévy associé à $\varphi(t,$.$) est à variation$ bornée. Nous utiliserons pour cela le critère donné par Bertoin ([1], p. 192) : il suffit d'établir que l'on a :

$$
\varlimsup_{\omega \rightarrow+\infty} \frac{\varphi(t, \omega)}{\omega}<+\infty
$$

Or, en effectuant le changement de variables $\omega \mapsto h(l, \omega)$, on vérifie facilement que :

$$
\varlimsup_{\omega \rightarrow+\infty} \frac{\varphi(t, \omega)}{\omega}=\varlimsup_{\omega \rightarrow+\infty} \frac{\varphi_{0}(h(t, \omega))}{\omega}=\lim _{\omega \rightarrow+\infty} \frac{\varphi_{0}(\omega)}{\omega+t \varphi_{0}(\omega)} \leq \frac{1}{t}<+\infty
$$

ce qui achève la preuve.

Tirons quelques conséquences faciles de ce théorème.

COROllaire 3. - Sous les conditions d'application du théorème 3, si pour tout $t>0, \varphi(t,$.$) admet la décomposition de Lévy-Khintchine (29) de la$ forme :

$$
\varphi(t, \omega)=b_{t} \omega+\int_{]-\infty, 0[}\left(e^{s \omega}-1\right) n_{t}(d s)
$$

Alors, pour $t>0$, le coefficient de dérive $b_{t}$ est donné par les formules :

$$
\left\{\begin{aligned}
b_{t}= & \frac{1}{t} \text { si } X(0) n^{\prime} \text { est pas à variation bornée } \\
b_{t}= & \frac{b_{0}}{1+t b_{0}} \text { si } X(0) \text { est à variation bornée } \\
& \text { de coefficient de dérive } b_{0}
\end{aligned}\right.
$$


Nous utiliserons pour la démonstration le lemme très élémentaire suivant :

LEMME 11. - Si la décomposition de Lévy-Khintchine du second exposant de Lévy d'un processus de Lévy homogène de variance finie, à sauts négatifs, à variation bornée est de la forme :

$$
\varphi(\omega)=b \omega+\int_{]-\infty, 0[}\left(e^{s \omega}-1\right) n(d s),
$$

alors, le coefficient de dérive b est donné par : $b=\lim _{\omega \rightarrow+\infty} \varphi^{\prime}(\omega)$.

Preuve du corollaire 3. - Il s'agit de déterminer la limite en $+\infty$ de la dérivée $\partial_{\omega} \varphi(t, \omega)$, laquelle concide, en vertu de la formule (35) avec :

$$
\frac{\varphi_{0}^{\prime}(h(t, \omega))}{1+\varphi_{0}^{\prime}(h(t, \omega))}
$$

Or $h(t,$.$) tend vers +\infty$ en $+\infty$ et la fonction $\varphi_{0}^{\prime}$ est croissante, donc a une limite $l_{0}$ en $+\infty$. Ainsi,

$$
b_{t}=\frac{l_{0}}{l+t l_{0}}
$$

De plus, d'après la décomposition (27) de $\varphi_{0}$, on a :

$$
\varphi_{0}^{\prime}(\omega)=a_{0}^{2} \omega+\hat{b}_{0}+\int_{]-\infty, 0[}\left(e^{s \omega}-1\right) s n_{0}(d s)
$$

d'où l'on déduit facilement que $l_{0}=a_{0}^{2} \infty+\hat{b}_{0}-\int_{]-\infty, 0[} s n_{0}(d s)$.

Le résultat annoncé est alors établi si l'on remarque de plus que lorsque $X(0)$ est à variation bornée, on $\mathbf{a}$ :

$$
b_{0}=\hat{b}_{0}-\int_{]-\infty, 0[} s n_{0}(d s) .
$$

-THÉREMe 4. - On se place dans les conditions d'application du théorème 3 et on note $X(t)$ le processus de Lévy associé au second exposant $\varphi(t,$.$) . Alors, avec une probabilité 1$, la fonction $x \mapsto X(t)(x)$ est dérivable presque partout, de dérivée $b_{t}$ donnée par la formule (38).

Afin de démontrer ce théorème, rappelons le résultat suivant :

Proposition 2 ([4], p. 316). - Soit $X$ un processus de Lévy homogène de variance finie et à sauts négatifs, de second exposant de Lévy : $\varphi(\omega)=$ $\int_{]-\infty, 0[}\left(e^{s \omega}-1\right) n(d s)$.

Alors,

$$
P\left(\lim _{\substack{a \rightarrow-\infty \\ b \rightarrow+\infty}} \frac{X(x)-X(0)}{x}=0\right)=1
$$

Vol. $15, \mathrm{n}^{\circ} 4-1998$. 
qui admet comme corollaire immédiat :

Corollaire 4. - Soit $X$ un processus de Lévy homogène de variance finie et à sauts négatifs, de second exposant de Lévy : $\varphi(\omega)=b \omega+\int_{]-\infty, 0[}\left(e^{s \omega}-\right.$ 1) $n(d s)$.

Alors, avec probabilité 1 , la fonction : $x \mapsto X(x)$ est dérivable presque partout, de dérivée $b$.

Le théorème 4 est donc une simple conséquence des corollaires 3 et 4 .

\section{LA CONDITION INITIALE BROWNIENNE}

Cette condition initiale a été particulièrement étudiée par Sinai [8] en utilisant la transformation de Hopf-Cole. De façon précise, il examine le cas où la condition initiale $X(0)$ vérifie :

$$
\left\{\begin{array}{l}
X(0)(x)=0 \quad \text { si } \quad x<0 \\
X(0)(x)=B(x) \quad \text { si } \quad x \geq 0
\end{array}\right.
$$

où $B$ est un mouvement brownien standard.

Rappelons très rapidement en quoi consiste la méthode de Hopf-Cole. L'équation de Burgers visqueuse :

$$
\partial_{t} X+\partial_{x}\left(\frac{1}{2} X^{2}\right)=\varepsilon \partial_{x}^{2} X
$$

est résolue explicitement en faisant le changement $X=-2 \varepsilon \frac{\partial_{x} Y}{Y}$ car la fonction $Y$ vérifie alors l'équation de la chaleur. La solution de l'équation non visqueuse est obtenue en faisant tendre le paramètre $\varepsilon$ de viscosité vers 0 ; ce qui met en cuvre une méthode de Laplace. La solution s'exprime à l'aide de la transformée de Legendre de la primitive de la condition initiale à laquelle est ajoutée une dérive linéaire.

Pour la condition initiale (39), Sinai est donc mené à étudier l'enveloppe convexe de la primitive du mouvement brownien avec dérive linéaire. Il obtient notamment l'analogue de notre théorème 4 , avec comme dérivée $\frac{1}{t}$.

Nous allons examiner ce même cas particulier en choisissant plutôt une condition initiale brownienne sur $\mathbb{R}$.

La condition initiale brownienne correspond au cas où $\varphi_{0}(\omega)=\frac{a^{2}}{2} \omega^{2}$. Il est alors facile de résoudre explicitement l'équation (31) à l'aide de la formule (34); on obtient ainsi :

$$
\varphi(t, \omega)=\frac{1+a^{2} \omega t \sqrt{1+2 a^{2} \omega t}}{a^{2} t^{2}} .
$$


La décomposition de Lévy-Khintchine (29) de la fonction $\varphi$ est de la forme :

$$
\varphi(t, \omega)=b_{t} \omega+\int_{]-\infty, 0[}\left(e^{s \omega}-1\right) n_{t}(d s)
$$

et on sait déjà avec la proposition 2 , que $b_{t}=\frac{1}{t}$. Des calculs élémentaires mènent à :

$$
\partial_{\omega}^{2} \varphi(t, \omega)=\frac{a^{2}}{\left(1+2 a^{2} t \omega\right)^{3 / 2}}=\int_{-\infty}^{0} e^{\omega s} \frac{\sqrt{|s|}}{a \sqrt{2 \pi t^{3}}} \exp \left(\frac{2}{2 a^{2} t}\right) d s
$$

et la mesure de Lévy $n_{t}$ est donc donnée par :

$$
n_{t}(d s)=\frac{1}{a \sqrt{2 \pi t^{3}}|s|^{3 / 2}} \exp \left(\frac{2}{2 a^{2} t}\right) d s
$$

Notons pour terminer qu'il est également possible d'expliciter la densité des accroissements $X(t)(x+h)-X(t)(x)$ : on obtient en effet après inversion de la transformation de Laplace donnée par la formule (40) :

$$
\begin{aligned}
& P(X)(t(x+h)-X(t)(x) \in d u) \\
& =1_{]-\infty, \frac{h}{t}\right]}(u) \frac{h}{a \sqrt{2 \pi t^{3}}} \\
& \quad \times \exp \left[-\frac{u^{2}}{2 a^{2} t(h / t-u)}\right] \frac{1}{(h / t-u)^{3 / 2}} d u .
\end{aligned}
$$

\section{REMERCIEMENTS}

Les auteurs remercient P. Bertoin pour leur avoir signalé une erreur dans la partie 4 de ce travail.

\section{RÉFÉRENCES}

[1] P. Bertorn, Lévy processes, Cambridge University Press, 1996.

[2] P. BillingsLeY, Convergence of probability measures, J. Wiley \& Sons, 1968.

[3] L. Breiman, Probability, SIAM Classics in Applied Mathematics, 1992.

[4] I. I. GIHMAN, A. V. SKOROHOD, The theory of stochastic processes II, Springer-Verlag, 1975.

[5] E. Hopf, The partial differential equation $u_{t}+u u_{x}=\mu u_{x x}$, Commun. Pure Appl. Mech., Vol. 3, p. 201-230, 1950.

[6] P. D. LAX, Hyperbolic systems of conservation laws and the mathematical theory of shock waves, SIAM, 1973. 
[7] Z.-S. She, E. Aurell, U. Frisch, The inviscid Burgers equation with initial data of brownian type, Commun. Math. Phys., Vol. 148, 1992, p. 623-641.

[8] Ya G. Sinal, Statistics of shocks in solutions of inviscid Burgers equation, Commun. Math. Phys., Vol. 148, 1992, p. 601-621.

(Manuscrit reçu le 26 février 1996). 